\title{
Mode of Delivery in Pregnant Females with Fibroid Uterus Presenting in Spontaneous Labour
}

\section{Uzma Aziz, ${ }^{1}$ Madiha Afzal, ${ }^{2}$ Hadia Shabbir ${ }^{3}$}

\begin{abstract}
Objectives: To determine the mode of delivery of obstetric population with uterine fibroids presenting in spontaneous labor.

Methods: It was a Descriptive cross sectional study done at Obstetric unit of Arif Memorial teaching Hospital, from June 2019 to May 2020. 50 pregnant patients with uterine fibroids in spontaneous labor were included in the study with $95 \%$ confidence level and $5 \%$ margin of error.

Results: In our study, $62 \%(n=31)$ patients were between 20 -30 years of age with $38 \%(n=19)$ between $31-40$ years with Mean age and SD $25.63 \pm 2.89$ years. 68\% $(\mathrm{n}=32)$ patients were between $34-36$ weeks of gestation whereas 32\% $(n=18)$ were between 37-39 weeks with Mean gestational age and SD 29.66 $\pm 1.59 .66 \%(n=33)$ patients were Primigravida to Gravida 3 and 34\% $(n=17)$ were Gravida4 to Gravida 5. The frequency of emergency caesarean section in patients with uterine fibroids was $58 \%(n=29)$ whereas $42 \%(n=21)$ cases with uterine fibroids had normal vaginal delivery. The most frequent indication for emergency cesarean section was labor dystocia i.e. (41.37\%).

Conclusion: The frequency of emergency caesarean section amongst patients with uterine fibroids presenting in spontaneous labor is high particularly due to labor arrest. The number of fibroids has an impact on mode of delivery in these patients. Therefore each obstetric patient with uterine fibroids, must be evaluated thoroughly for the appropriate mode of delivery in prenatal period.

Key Words: Pregnancy, uterine fibroids, mode of delivery, cesarean section, spontaneous vaginal delivery

How to Cite: Aziz U, Afzal M, Shabbir H. Mode of delivery in pregnant females with fibroid uterus presenting in spontaneous labour. Esculapio.2020;16(04):74-78.
\end{abstract}

DOI: https://doi.org/10.51273/esc20.2516416

\section{Introduction}

$\mathrm{T}$ he fibroids are the benign tumors of smooth muscle cells of the uterus. They are the most frequent benign tumors found in females in their reproductive age. The incidence of the uterine fibroids increases to $40 \%-60 \%$ by 35 years of age and rises to $70 \%-80 \%$ at 50 years of age but still the etiology of uterine fibroids is not exactly understood. ${ }^{1}$

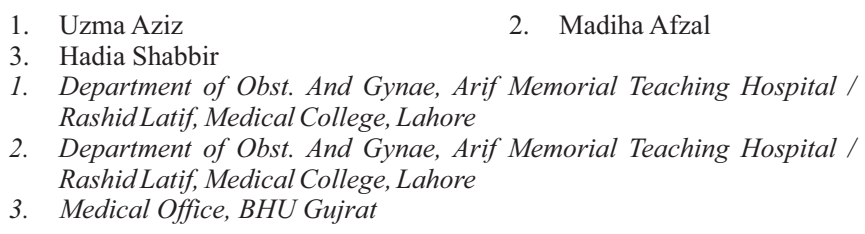

Dr. Uzma Aziz, Assistant Professor, Department of Obst. And Gynae, Arif Memorial Teaching Hospital / Rashid Latif, Medical College, Lahore E-mail: uzmaazizjaved@gmail.com

$\begin{array}{ll}\text { Submission Date: } & 11-09-2020 \\ \text { 1st Revision Date: } & 15-10-2020 \\ \text { Acceptance Date: } & 01-11-2020\end{array}$

The incidence of preg-nancy with fibroids is about $0.5 \%$ to $4 \%{ }^{2}$ The preva-lence of fibroids in pregnancy in Pakistan is $0.87 \%{ }^{3}$ The frequency of uterine fibroid with pregnancy is increasing because of couple's tendency to delay first pregnancy till the age of 30. Due to increase in estro-gen/ progestin levels, $15 \%-30 \%$ of fibroids will enlarge in pregnancy. However most of the fibroids shrink in size during puerperium. ${ }^{4}$ Less than $3 \mathrm{~cm}$ Intramural and sub serosal fibroids are not clinically significant. Pregnancies associated with uterine fib-roids are labelled as high risk pregnancies. Most of these pregnancies with fibroid uterus are usually uneventful but sometimes, serious complications can occur during the course of pregnancy, depending upon the size, site and location of the fibroid. ${ }^{5}$ About $10 \%-30 \%$ of females with uterine fibroids face obstetric complications during pregnancy. ${ }^{1}$ The recent evidence reports a higher rate of cesarean delivery 
particularly in women with large uterine fibroids when compared with normal vaginal delivery rate i.e. $48.8 \%$ vs. $13.3 \%$ and the most common cause for emergency caesarean section was dysfunctional labor while malpresentation was the commonest indication for elective caesarean section in these patients. ${ }^{6}$ Women with uterine fibroid have increased caesarean section rate due to distortion of uterine cavity or other obstetric reasons. ${ }^{4}$ In such pregnancies caesarean section rate, postpartum hemorrhage and prolong hospital stay is found to be high and needs specific follow up..$^{7-9}$ Conversely, Vergani et al. reported a high vaginal delivery rate amongst laboring women with fibroids and concluded that pregnant women with fibroids should be counseled for the trial of labor. ${ }^{10}$ Ciavattini et al. found high rate of emergency caesarean section in patients with multiple uterine fibroids as compared to pregnant females with single or no fibroids delivering vaginally. ${ }^{11}$ The size, number and location of uterine fibroids have significant impact on the mode of delivery. So prior information is useful in assigning risk category of a pregnant female with fibroids. ${ }^{12}$ Our study was aimed to determine the mode of deli-very in pregnant obstetric population with fibroid uterus presenting in spontaneous labour. In our study the mode of delivery of pregnancy with uterine fibroid was dependent on the size, number of fibroids that was comparable to the study of Poovathi and Ramalingam. ${ }^{13}$ The inconsistent available data in the literature about the preferred mode of delivery in pregnancies with uterine fibroids provides the reason to carry out this research. As pregnant females with uterine fibroids have greater concerns to know about their mode of delivery, our study will be a good addition in previous available literature to determine the mode of delivery in these patients thus making counseling of these patients to be done appropriately regarding mode of delivery.

\section{Methods}

It was Descriptive cross-sectional study which was conducted at Obstetric unit of Arif memorial teaching Hospital, Lahore from June 2019 to, May 2020 after taking approval from ethical committee of RLMC. 50 pregnant females with uterine fibroids having spontaneous labor were selected with a $95 \%$ Confidence level and $5 \%$ margin of error with non-probability purposive sampling technique after fulfilling the inclusion criteria. Written informed consent was taken from the patients to use their data for this study. The collected data was analyzed by using SPSS version 17.

\section{Following were Included}

- 20-40 years of age

- $\quad$ PG to Gravida 5

- with a Gestational age 34 weeks - 39 weeks

- $\quad$ single or multiple uterine fibroids $>3 \mathrm{~cm}$

- having spontaneous labor

\section{Following were Excluded}

- Pregnancy with maternal medical disorders

- Previous caesarean section

- Fetal malpresentation

- Placenta previa

- Cervical fibroids

- Inadequate pelvis

- Lower segment uterine fibroid of $>8 \mathrm{~cm}$ were excluded from the study.

All patients were given a trial of labor and followed till their delivery. Their progress of labor was monitored with partogram and fetal condition was assessed by continuous fetal heart rate monitoring with cardiotocography.

\section{Results}

A total of 50 cases fulfilling the study criteria were included to assess the rate of caesarean section in pregnant patients with uterine fibroids.

Age distribution of the patients showed that $62 \%$ $(\mathrm{n}=31)$ were between 20-30 years whereas 38\% \% $(n=19)$ were between 31-40 years of age with mean and standard deviation of the patient's age as $25.63 \pm$ 2.89 years. (Table 1). 68\% $(\mathrm{n}=32)$ patients were between $34+0-36+6$ weeks of gestation whereas $32 \%$ $(n=18)$ were between $37+0-39+6$ weeks while the mean and standard deviation for the gestation age was determined as 29.66 \pm 1.59 . (Table 1). $66 \%(n=33)$ patients were between Primigravida to Gravida3 and $34 \%(n=17)$ were between Gravida4 to Gravida5. (Table 1). The frequency of caesarean section in patients with uterine fibroids was $58 \% \quad(n=29)$ whereas $42 \%(n=21)$ cases with uterine fibroids had spontaneous vagi-nal delivery. (Table 2). The most frequent indication for emergency caesa-rean section was labor dystocia $12(41.37 \%)$ followed by fetal distress 10(34.50\%), PROM with poor bishop score $4(13.79 \%)$, cord prolapse $2(6.89 \%)$, and placental 
Table 1: Distribution of Age, Parity and Gestation Age

\begin{tabular}{lcc}
\hline \multicolumn{1}{c}{ Age(in years) } & No. of patients & Percentage \\
\hline $20-30$ & 31 & $62 \%$ \\
$31-40$ & 19 & $38 \%$ \\
Total & 50 & $100 \%$ \\
Mean \pm SD & $25.63 \pm 2.89$ & \\
Gestational Age in weeks & & \\
$34^{+0}-36^{+6}$ & 34 & $68 \%$ \\
$37^{+0}-39^{+6}$ & 16 & $32 \%$ \\
Total & 50 & $100 \%$ \\
Mean \pm SD & $29.66 \pm 1.59$ & \\
Parity & & \\
Primigravida-Gravida ${ }^{3}$ & 33 & $66 \%$ \\
Gravida ${ }^{4}$-Gravida & \\
Total & 17 & 34 \\
\hline
\end{tabular}

Table 2: Mode of delivery in patients with uterine fibroids

\begin{tabular}{lcc}
\hline \multicolumn{1}{c}{ MOD } & No. of patients & Percentage \\
\hline Caesarean section & 29 & $58 \%$ \\
Spontaneous vaginal delivery & 21 & $42 \%$ \\
Total & 50 & $100 \%$ \\
\hline
\end{tabular}

Table 3: Indication for emergency Caesarean section in patients with fibroids

\begin{tabular}{lcc}
\hline \multicolumn{1}{c}{ Indication } & No. of Patients & Percentage \\
\hline Labor Dystocia & 12 & $41.37 \%$ \\
Fetal Distress & 10 & $34.50 \%$ \\
PROM with poor bishop score & 4 & $13.79 \%$ \\
Cord Prolapse & 2 & $6.89 \%$ \\
Placental Abruption & 1 & $3.44 \%$ \\
Total & 29 & $100 \%$ \\
\hline
\end{tabular}

abruption $1(3.44 \%)$ being the least common indication for abdominal delivery. (Table 3)

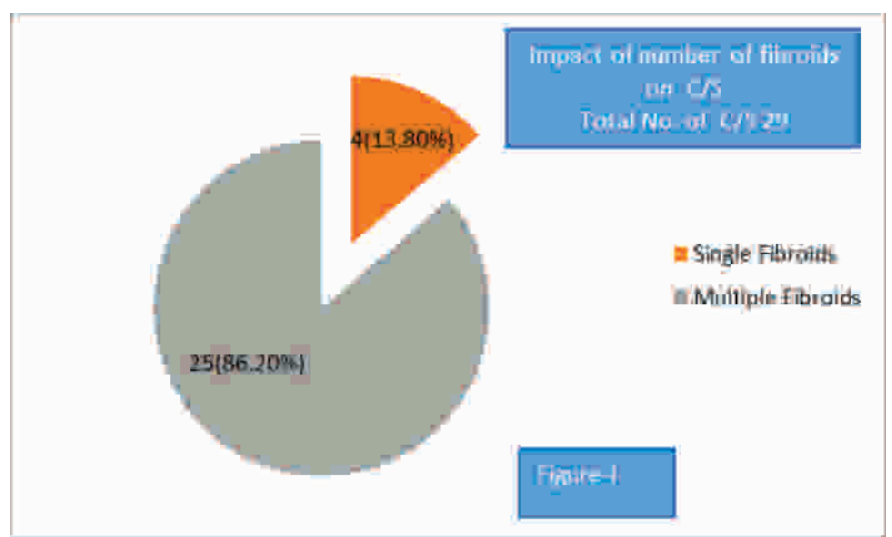

Figure 1: shows that 25(86.20\%) patients with multiple fibroids having trial of labor ended up in emergency caesarean section as compared to $4(13.80 \%)$ patients with single fibroid who had caesarean section.

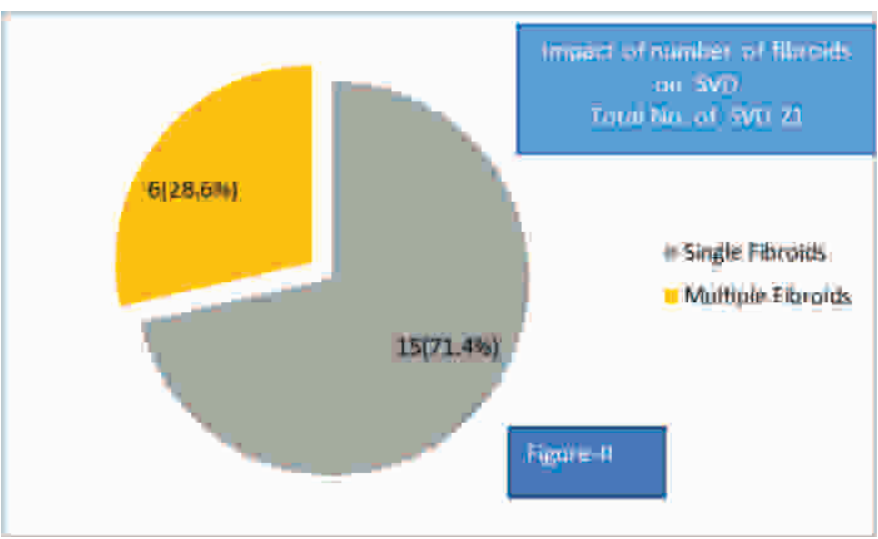

Figure 2: shows that $15(71.4 \%)$ patients with single fibroid delivered vaginally when compared to 6 $(28.6 \%)$ patients with multiple fibroids delivering vaginally.

\section{Discussion}

Uterine fibroids are the commonest benign tumors of the female genital tract. They occur in 30-70\% females in their reproductive ages. ${ }^{14}$ These tumors have been implicated as a risk factor for increased caesarean section in pregnant women. The frequency of fibroids increases with the advancing age of the females and is generally most common among African American females. ${ }^{15}$ The prevalence of uterine fibroid in pregnancy is $0.1 \%-10.7 \%{ }^{16,17}$ Presence of fibroids in pregnancy increases the likelihood of obstetric complication by $10 \%-30 \%$ while increasing the frequency of caesarean section up to $70 \% .{ }^{18}$ Past studies on delivery outcomes in females with uterine fibroids have shown disparities and inconsistent results. These studies show that in such pregnancies, the incidence of operative delivery increases. ${ }^{19,}{ }^{20} \mathrm{In}$ our study, $62 \%(n=31)$ of the patients were bet-ween 20-30 years of age. This finding is in compa-rison to the study of Javed M who concluded that $59 \%$ of the pregnant females with fibroid uterus presented at a younger age i.e. between 20-30 years of age. ${ }^{5}$ The increased incidence of uterine fibroids in young pregnant obstetric population can be justified by quoting that estrogen and progesterone which stimulate the uterine lining during menstrual cycle in preparation for pregnancy appear to promote the growth of fibroids which contain more estrogen and progesterone receptors than normal uterine muscles do. In our study, $66 \%(n=33)$ patients were between para 1 to para 3 and $34 \%(n=17)$ were between para 4 to para 5 . The fibroids are usually associated with nulliparity. The relative risk of fibroids decreases with increasing parity and each additional term pregnancy, the risk is reduced to one fifth with five term pregnancies compared with nulliparous females. ${ }^{21}$ This is similar to the findings in nulliparous black females in whom 
there is a nine-fold increase in the incidence of fibroids. Females with uterine fibroids have had fewer term pregnancies and are generally of lower parity than the females without this problem. ${ }^{22}$ The frequency of caesarean section in our patients with uterine fibroids was $58 \%(n=29)$ and $42 \%(n=21)$ patients with uterine fibroids had a normal vagi-nal birth. The results of the current research are in concurrence with the study done by Javed $\mathrm{M}$ indicating that the caesarean delivery rate was $63.5 \%$ in pregnancies with fibroid uterus. ${ }^{5}$ One research shows that there is the strong association between the uterine fibroids and an increased risk of caesarean section, particularly with large size fibroid. Another study by Youssef A, recorded the caesarean section in 47.8\% of the pregnant patients with fibroids which is less than that of our results. ${ }^{23}$ The increased rate of emergency caesarean section in our study was noticed because of increased incidence of dysfunctional labor due to distorted uterine anatomy leading to failure to progress and large tumor volume. Also the most common indication for emergency caesarean section in our patients was labor dystocia i.e. 12 patients $(41.37 \%)$, followed by fetal distress i.e. 10 (34.5\%) patients, PROM with poor BISHOP score i.e. 4 (13.7\%) patients, Cord prolapse i.e. $2(6.8 \%)$ patients and placental abruption i.e. $1(3.5 \%)$ patients. This finding of our study was comparable with the research done by NOOR S showing that failure to progress (39\%) and fetal distress (38\%), PROM $(14 \%)$ were the most common indications and placental abruption (3.3\%) the least common indication for emergency caesarean delivery. ${ }^{24}$ Although previous literature and our study reports that uterine fibroids are associated with an increased rate of emergency caesarean section in patients having trial of labor. ${ }^{25,26}$ We also determined the association between the number of fibroids and its impact on mode of delivery thus observing that our study population having multiple fibroids ended up more in emergency caesarean section than those having single fibroid who successfully ended in having vaginal delivery i.e. $(86 \%$ vs. $71 \%)$. This finding is in comparison to the study of Zhao R who concluded that SVD rate is high in patients with single fibroids than patients with multiple fibroids. ${ }^{12}$ However Valerie I et al didn't find difference in the mode of delivery (Normal vaginal Delivery and caesarean section) in females with multiple fibroids compared with those having single or no fibroids. ${ }^{27}$ This finding was also supported by Stout et al in which females with single fibroid $5<\mathrm{cm}$ didn't have an increased risk of a caesarean delivery when contrasted with females with multiple fibroids $>5 \mathrm{~cm}^{28}$ The limitation of our study was that we didn't consi-der the location of the fibroids (subserosal, intramural or submucosal) to see the impact on mode of delivery.

\section{Conclusions}

We concluded from our study that the rate of emergency caesarean section is higher among patients with uterine fibroids with labor dystocia the most common indication of emergency caesarean section. So it is recommended that

1. Each patient who presents with uterine fibroids, must be assessed at consultant level at term to determine the suitable mode of delivery.

2. Promote obstetric practice that multiple/ large fibroids are not absolute contraindication for trial of labor provided prior assessment of patient has been done in antenatal period to be eligible for trial of labor.

These strategies will help to reduce the frequency of caesarean section in pregnant patients with fibroid uterus thus increasing chances of normal vaginal delivery.

\section{Conflict of Interest and Funding:}

None

\section{References}

1. Lee HJ, Norwitz ER, Shaw J. Contemporary management of fibroids in pregnancy. Reviews in Obstetrics and Gynecology. 2010;3(1):20.

2. Tchente NC, Fogaing A, Tejiokem M, Nana NP, Mbu R, Leke R. Pregnancy outcome in a group of Cameroonian women with uterine fibroids. Journal de gynecologie, obstetrique et biologie de la reproduction. 2009;38(6):493.

3. Egbe TO, Badjang TG, Tchounzou R, Egbe E-N, Ngowe MN. Uterine fibroids in pregnancy: prevalence, clinical presentation, associated factors and outcomes at the Limbe and Buea Regional Hospitals, Cameroon: a cross-sectional study. BMC research notes. 2018;11(1):889.

4. Ortiz FM, Romero BP, García EE, Barraza JB, Castro EQ, Garay FdJP. Uterine leiomyomas during pregnancy and its impact on obstetric outcome. Ginecologia y obstetricia de Mexico. 2011;79(08):467-73.

5. JAVED M, TARIQ R, RASHID M. Effect of Uterine Fibroid on Pregnancy Outcome. Pain (Abdominal pain).PJMHS.2010;4(4):476-478.

6. Qidwai GI, Caughey AB, Jacoby AF. Obstetric outcomes in women with sonographically identified uterine leiomyomata. Obstetrics \& Gynecology. 2006; 107(2): 376-82.

7. Klatsky PC, Tran ND, Caughey AB, Fujimoto VY. Fibroids and reproductive outcomes: a systematic literature review from conception to delivery. American journal of obstetrics and gynecology. 2008; 198(4): 357-66.

8. Lam S-J, Best S, Kumar S. The impact of fibroid 
characteristics on pregnancy outcome. American journal of obstetrics and gynecology. 2014; 211(4): 395. e1-.e5.

9. Sunkara SK, Khairy M, El-Toukhy T, Khalaf Y, Coomarasamy A. The effect of intramural fibroids without uterine cavity involvement on the outcome of IVF treatment: a systematic review and meta-analysis. Human Reproduction. 2010;25(2):418-29.

10. Vergani P, Locatelli A, Ghidini A, Andreani M, Sala F, Pezzullo JC. Large uterine leiomyomata and risk of cesarean delivery. Obstetrics \& Gynecology. 2007; 109(2): 410-4.

11. Ciavattini A, Clemente N, Delli Carpini G, Di Giuseppe J, Giannubilo SR, Tranquilli AL. Number and size of uterine fibroids and obstetric outcomes. The journal of maternal-fetal \& neonatal medicine. 2015;28(4):484-8.

12. Zhao R, Wang X, Zou L, Li G, Chen Y, Li C, et al. Adverse obstetric outcomes in pregnant women with uterine fibroids in China: A multicenter survey involving 112,403 deliveries. Plos one. 2017; 12(11): e0187821.

13. Poovathi M, Ramalingam R. Maternal and Fetal Outcome in Pregnancy with Fibroids: A Prosp ective Study. International Journal of Scientific Study. 2016;3(11):169-72.

14. Okolo S. Incidence, aetiology and epidemiology of uterine fibroids. Best practice \& research Clinical obstetrics \& gynaecology. 2008;22(4):571-88.

15. Marshall LM, Spiegelman D, Barbieri RL, Goldman MB, Manson JE, Colditz GA, et al. Variation in the incidence of uterine leiomyoma among premenopausal women by age and race. Obstetrics \& Gynecology. 1997;90(6):967-73.

16. Hasan F, Arumugam K, Sivanesaratnam V. Uterine leiomyomata in pregnancy. International Journal of Gynecology \& Obstetrics. 1991;34(1):45-8.

17. Laughlin SK, Baird DD, Savitz DA, Herring AH, Hartmann KE. Prevalence of uterine leiomyomas in the first trimester of pregnancy: an ultrasound screening study. Obstetrics and gynecology. 2009; 113(3): 630.

18. Katz VL, Dotters DJ, Droegemeuller W. Complications of uterine leiomyomas in pregnancy. Obstetrics and gynecology. 1989;73(4):593-6.

19. Coronado GD, Marshall LM, Schwartz SM. Compli- cations in pregnancy, labor, and delivery with uterine leiomyomas: a population-based study. Obstetrics \& Gynecology. 2000;95(5):764-9.

20. Michels KA, Edwards DRV, Baird DD, Savitz DA, Hartmann KE. Uterine leiomyomata and cesarean birth risk: a prospective cohort with standardized imaging. Annals of epidemiology. 2014;24(2):122-6.

21. Vollenhoven B, Lawrence A, Healy D. Uterine fibroids: a clinical review. BJOG: An International Journal of Obstetrics \& Gynaecology. 1990;97(4):28598.

22. JAVED M, SHAHID A, TARIQ S. EFFECT OF UTERINE FIBROID ON MODE OF DELIVERY. Pakistan Postgraduate Medical Journal. 2016; 27(2): 40-3.

23. Youssef A, Aissia B, Gara M. Association fibromyoma and pregnancy. About 23 cases. La Tunisie Medicale. 2005;83(4):194-7.

24. Noor S, Fawwad A, Sultana R, Bashir R, Jalil H, Suleman N, et al. Pregnancy with fibroids and its obstetric complications. Journal of Ayub Medical College Abbottabad. 2009;21(4):37-40.

25. Rice JP, Kay HH, Mahony BS. The clinical significance of uterine leiomyomas in pregnancy. American journal of obstetrics and gynecology. 1989; 160(5): 1212-6.

26. Sheiner E, Bashiri A, Levy A, Hershkovits R, Katz M, Mazor M. Obstetric characteristics and perinatal outcome of pregnancies with uterine leiomyomas. Obstetrical \& gynecological survey. 2004;59(9):6478.

27. Shavell VI, Thakur M, Sawant A, Kruger ML, Jones TB, Singh M, et al. Adverse obstetric outcomes associated with sonographically identified large uterine fibroids. Fertility and sterility. 2012;97(1):107-10.

28. Stout MJ, Odibo AO, Graseck AS, Macones GA, Crane JP, Cahill AG. Leiomyomas at routine secondtrimester ultrasound examination and adverse obstetric outcomes. Obstetrics \& Gynecology. 2010; 116 (5): 1056-63.

\section{Authors Contribution}

AU: Literature Search, Study Concept \& Design

AM: Data Collection

SH: Data Collection and Analysis 